\title{
The magnetic structure of an earthward-moving flux rope observed by Cluster in the near-tail
}

\author{
Y. C. Zhang ${ }^{1,2}$, Z. X. Liu ${ }^{1}$, C. Shen ${ }^{1}$, A. Fazakerley ${ }^{3}$, M. Dunlop ${ }^{4}$, H. Réme ${ }^{5}$, E. Lucek ${ }^{6}$, A. P. Walsh ${ }^{3}$, and L. Yao ${ }^{1,2}$ \\ ${ }^{1}$ State Key Laboratory of Space Weather, Center for Space Science and Applied Research, Chinese Academy of Sciences, \\ Beijing 100080, China \\ ${ }^{2}$ Graduate School of the Chinese Academy of Sciences, Beijing 100080, China \\ ${ }^{3}$ Mullard Space Science Laboratory, Univ. College London, London, UK \\ ${ }^{4}$ Rutherford Appleton Laboratory, Chilton, DIDCOT, Oxfordshire OX11 0QX, UK \\ ${ }^{5}$ Centre d'Etudes Spatiale des Rayonnements, BP 4346, 31028 Toulouse Cedex 4, France \\ ${ }^{6}$ SAPG, Imperial College London SW7 2BZ, UK
}

Received: 11 April 2007 - Revised: 12 June 2007 - Accepted: 18 June 2007 - Published: 30 July 2007

\begin{abstract}
We investigate the magnetic structure of a small earthward-moving flux rope observed by Cluster in the near-Earth plasma sheet through application of the GradShafranov (GS) technique to reconstruct the transverse magnetic field distribution perpendicular to the flux rope axis at $\mathrm{X}=-14.75 R_{E}$. We find that the principal axis of the flux rope lies approximately along the dawn-dusk direction and that the diameter of the flux rope is about $1.5 R_{E}$. There is a strong duskward core magnetic field in the center of the flux rope. According to the AE index, there is no obvious substorm associated with the magnetic flux rope. Recent studies indicate that the formation of the flux rope in the plasma sheet can be understood in terms of simultaneous reconnection at multiple $\mathrm{X}$-line points in the near-tail. The distribution of the transverse magnetic field on the cross section is the asymmetric circles, which requires that the reconnections at multiple $\mathrm{X}$-line points occur. So our results also provide additional evidence for the occurrence of multiple- $X$ line reconnection in the magnetotail.
\end{abstract}

Keywords. Magnetospheric physics (Magnetotail; Plasma sheet) - Space plasma physics (Experimental and mathematical techniques; Magnetic reconnection)

\section{Introduction}

The magnetic flux rope is a common and very important physical phenomenon which occurs throughout the Earth's magnetosphere and interplanetary space. When an east-west oriented magnetic flux rope in the magnetotail passes by a

Correspondence to: Y. C. Zhang

(zyc@ cssar.ac.cn) satellite, characteristic north-then-south or south-then-north $B_{z}$ bipolar signals, together with high speed flows, are observed: if the motion is toward (away from) the Earth, the $B_{z}$ bipolar signals will have south-then-north (north-thensouth) polarity. Hughes and Sibeck (1987) first pointed out that the magnetic flux rope should be described as a threedimensional "rope" which has a helical magnetic field structure together with a strong core field, and arises from the shear between the magnetic fields in the northern and southern plasma sheet. In earlier studies, more attention has been paid to magnetic flux ropes in the distant magnetotail at $X<-60 R_{E}$ (e.g. Kivelson et al., 1993; Moldwin et al., 1993). The launch of more and more satellites covering other regions of the magnetotail, such as Geotail, Cluster and Double Star, has allowed observation of magnetic flux ropes in the near-Earth magnetotail, at $\mathrm{X}>-30 R_{E}$ (Elphic et al., 1986; Nishida et al., 1986; Slavin et al., 2003a, 2003b; Zhang et al., 2007). Slavin et al. (2003a) argued that nearEarth magnetic flux ropes are the result of near-Earth magnetic reconnection at multiple X-line points in a similar manner to the formation of FTEs at the dayside magnetopause (Lee, 1995). The field tension or the plasma jet produced by lobe field reconnection pushes the flux rope earthward or tailward. In the opinion of Slavin, magnetic flux ropes can be regarded as the preliminary event to the onset of open field reconnection in the NENL model of the substorm (Baker et al., 1996).

The ideal model of a magnetic flux rope is based on the bipolar magnetic signature and the strong core field typically observed by satellites crossing the real magnetic flux rope. To illustrate the geometry, Fig. 1 shows the typical magnetic field signature in a flux rope based on the ideal model (Lepping et al., 1990). The line labelled " 1 " is the

Published by Copernicus Publications on behalf of the European Geosciences Union. 

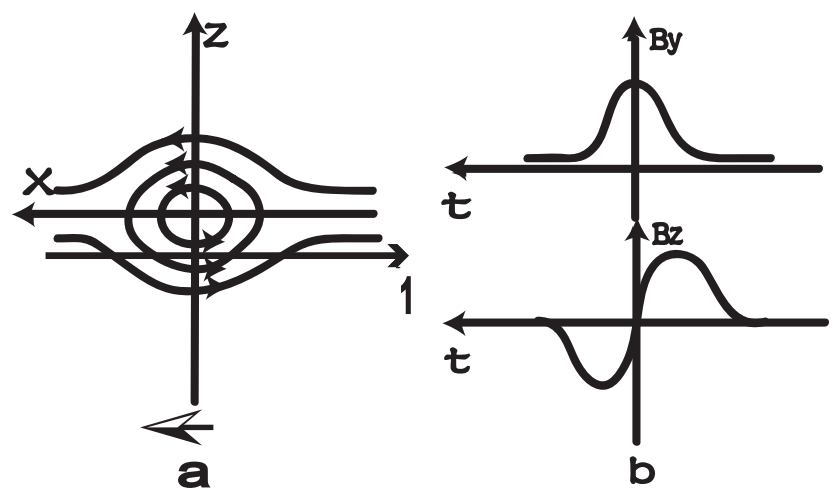

Fig. 1. Illustration of the magnetic signature in the magnetic flux rope. Panel (a) demonstrates the cross section of an earthward moving magnetic flux rope. Panel (b) displays the magnetic field components observed by satellite along line 1 .

imagined trajectory of the satellite crossing the magnetic flux rope. Figure 1a demonstrates the cross section of an earthward moving magnetic flux rope and Fig. 1b displays the magnetic field components observed by the satellite along line 1. At the present time, there is still no description of the complete configuration of the magnetic field in a real magnetic flux rope in the magnetotail. Nevertheless, using the four-point magnetic measurements of Cluster, however, Shen et al. (2007) have developed a magnetic rotation analysis (MRA) approach which can investigate the geometrical structure of the magnetotail flux rope. They find that, the deduced magnetic structure of one near-Earth flux rope is in agreement with the empirical model (Elphic and Russell, 1983) and that its characteristic spatial scale is about $1 R_{E}$.

The Grad-Shafranov technique (Hau and Sonnerup, 1999) has been used to study the magnetic structure of the magnetopause (Hasegawa et al., 2004, 2005), of magnetic clouds in the interplanetary medium (Hu and Sonnerup, 2002) and of FTEs at the magnetopause (Sonnerup et al., 2004). For quasi-two-dimensional structures, with the help of this technique, one can compute the magnetic field distribution of the transverse section perpendicular to the invariant axis of the object studied. The magnetic flux rope in the magnetotail has the same observational character as FTEs and flux ropes associated with interplanetary magnetic clouds. This allows us to use here the Grad-Shafranov technique to reconstruct the magnetic flux rope observed by Cluster at the magnetotail at $\mathrm{X}=-14.75 R_{E}$. We highlight a particularly stable event which allows accurate parameters to be computed.

\section{Observation}

On 11 October 2003, the four Cluster spacecrafts were operating in an elliptical polar orbit, with apogee in the magnetotail. At 07:40 UT, the satellites were located at (-14.75, $8.96,1.96) R_{E}$. The FGM (Balogh et al., 2001), CIS (Rème et al., 2001) and PEACE (Johnstone et al., 1997) experiments on board the satellites, respectively, provided 4-s averaged magnetic field data, ion data and electron data in the interval surrounding this time. Figure 2 shows the Cluster observations from 07:43 to 07:53 UT in GSE coordinates. From top to bottom of Fig. 2, the following parameters are plotted: electron density $\left(N_{e}\right)$, electron temperature $\left(T_{e}\right)$, electron thermal pressure $\left(P_{e}\right)$, ion density $\left(N_{i}\right)$, ion temperature $\left(T_{i}\right)$, ion thermal pressure $\left(P_{i}\right)$, ion plasma beta $\left(\beta_{i}\right)$, ion velocity components $\left(V_{x}, V_{y}, V_{z}\right)$ and speed $\left(V_{t}\right)$, magnetic field components $\left(B_{x}, B_{y}, B_{z}\right)$ and total magnetic intensity $\left(B_{t}\right)$. The implication of $B_{x}<0$ places Cluster in the Southern Hemisphere and a temperature $T_{i}=4 \mathrm{keV}$ indicates that Cluster is in the southern plasma sheet at this time. Based on the $B_{z}$ signature, two vertical solid lines mark the boundary of the flux rope to be empirically reconstructed in our analysis. As can be seen from the $B_{z}$ bipolar signal, at 07:46:47 UT Cluster enters the flux rope from the plasma sheet. $B_{z}$ decreases from $1.22 \mathrm{nT}$ to $-7.8 \mathrm{nT}$ and then increases to $7.1 \mathrm{nT}$. The peak-to-peak value of $B_{z}$ is $14.9 \mathrm{nT}$. Along with this $B_{z}$ variation, there is a $\left|B_{y}\right|$ enhancement, increasing from $4 \mathrm{nT}$ to $12 \mathrm{nT}$. Furthermore, the $B_{y}$ enhancement leads to a peak in total magnetic field intensity, which is 2.5 times greater than the adjacent plasma sheet magnetic field. Cluster exits the flux rope at 07:47:52 UT and then $B_{y}$ and $B_{z}$ restore their original values in the plasma sheet.

Slavin et al. (2003a) point out that the flux ropes observed by Geotail are usually accompanied by high speed flows and explain the fact that the ion density in the flux rope is higher than the value in the adjacent plasma sheet as due to compression between the flux rope and the plasma ahead of it. Their interpretation is that after the formation of the flux rope by multiple $\mathrm{X}$-line magnetic reconnection, one of the $\mathrm{X}$-lines in the plasma sheet first reconnects the open lobe fields inevitably (Schindler, 1974) to trigger the substorm. Then the flux rope will move toward the Earth or toward the tail under the push of field tension or the bulk flow produced by lobe field reconnection. The plasma in our case, however, does not show a character different from the adjacent plasma sheet: no high speed flow and high ion density are observed. The AE index is quiet, suggesting that no substorm occurs in the interval corresponding to this case; therefore, according to the NENL model of substorm (Baker et al., 1996), we can infer that there is no lobe field reconnection after the formation of this flux rope. Thus, it is understandable that there is no high speed flow in the case here. The observations of this flux rope may suggest that not all multiple X-line magnetic reconnection at the tail will lead to severe reconnection of the lobe fields, as argued by Schindler (1974) as a trigger for substorms. 


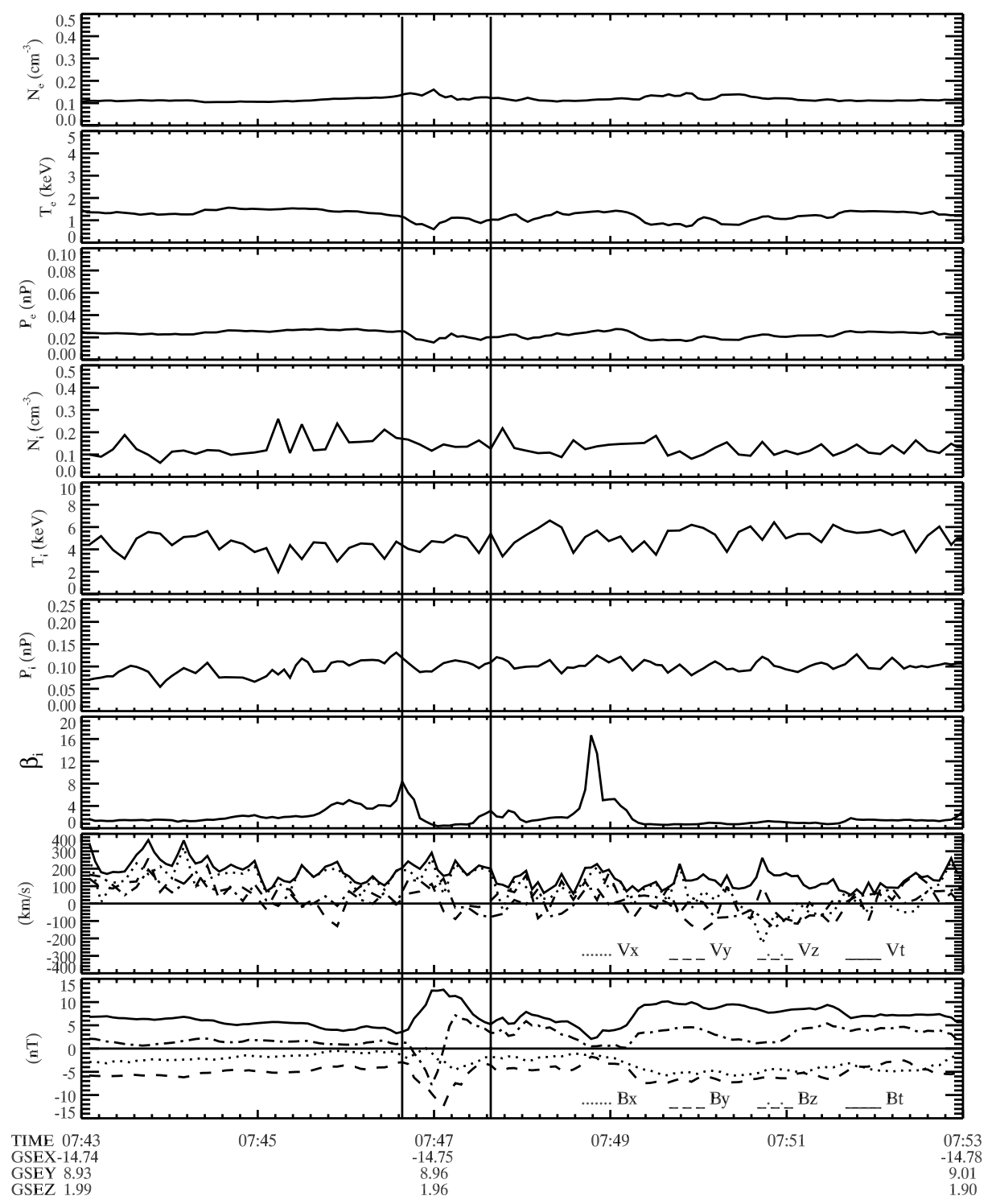

Fig. 2. Cluster observation of the flux rope from 07:43 UT to 07:53 UT on 11 October 2003 in the GSE coordinates. From the top to the bottom of the Fig. 2, the following parameters are listed: electron density, electron temperature, electron thermal pressure, ion density, ion temperature, ion thermal pressure, ion plasma beta, ion velocity components and speed, magnetic field components and total intensity.

\section{Grad-Shafranov method}

The GS reconstruction method (Hau and Sonnerup, 1999) is based on the following three assumptions:

1. The object to be reconstructed should have approximately a 2.5 -dimension structure, i.e. the spatial variation in one direction (invariant $\mathrm{Z}$ axis of the object) should be much slower than those at the transverse section (X-Y plane) perpendicular to that direction $(\partial / \partial z \approx 0)$. Thus, the emphasis of our study can be focused on the physical parameters in the X-Y plane.
2. A frame must exist, usually the deHoffmann-Teller frame (HT frame) (deHoffmann and Teller, 1950), in which the object to be studied is approximately temporally stationary $(\partial / \partial t \approx 0)$. This HT frame moves with the velocity $\boldsymbol{V}_{H T}$ relative the satellite.

3. In the HT frame, the inertial effect of the plasma can be neglected. If the plasma velocity in the HT frame is much smaller than the Alfvén speed and the acoustic speed, this assumption can be satisfied.

Under the above three assumptions the equation of motion of the plasmas in $\mathrm{X}-\mathrm{Y}$ plane can be expressed as

$\nabla_{t} P=(\boldsymbol{J} \times \boldsymbol{B})_{t}$. 
Here the $\mathrm{X}-\mathrm{Y}$ plane is the transverse cross section in the reconstruction coordinate (the orthogonal coordinate determined from the invariant $\mathrm{Z}$ axis and the direction of $-\boldsymbol{V}_{H T}$ ). The subscript " $t$ " denotes the transverse components of the parameter in the XY plane.

The magnetic vector potential $\boldsymbol{A}=A(x, y) \boldsymbol{z}$ can be introduced. Under the assumption of $\partial / \partial z \approx 0$, the magnetic field vector is expressed as

$\boldsymbol{B}=\left[\partial A / \partial y,-\partial A / \partial x, B_{z}(A)\right]$.

With the Biot-Savart law and Eq. (2), the Eq. (1) reduces to the Grad-Shafranov (GS) equation:

$\nabla_{t}^{2} A=-\mu_{0} \frac{d}{d A} P_{t}(A)$,

where $P_{t}=P+B_{z}^{2} / 2 \mu_{0}, P$ is the plasma's thermal pressure and $B_{z}^{2} / 2 \mu_{0}$ is the magnetic pressure of the axis field component. Both $P$ and $B_{z}$ are the functions of A alone. The magnetic field and plasma data measured by the Cluster array when it crosses the structure are taken as the initial values required to solve the GS equation and obtain the distribution of magnetic potential $A$ (magnetic field $\boldsymbol{B}$ ) in the XY plane. From Eq. (2) we can deduce $\cdot \nabla A=0$, so the field line in the flux rope is the contour line of A. The details of the GS method have been given by Hau et al. (1999) and Hu et al. (2002).

The GS method requires that the satellite crosses the structure and measures the components of the magnetic field, the components of the plasmas velocity, the temperature and density of the plasma. The next steps compute the reconstruction:

1) Determine the HT frame velocity from the magnetic field and the plasma data, measured by minimizing the residual electric field (Khrabrov and Sonnerup, 1998). In the HT frame the electric field almost vanishes, so from the equation $\nabla \times \boldsymbol{E}=\partial \boldsymbol{B} / \partial t$ we have $\partial \boldsymbol{B} / \partial t=0$. That is to say the magnetic structure in the flux rope is stationary. If the plasma velocity in the HT frame is small enough to be neglected, we may regard that the satellite crosses the magnetic flux rope approximately with the velocity $-\boldsymbol{V}_{\boldsymbol{H} T}$.

2) Find a working frame $\left(x^{\prime}, y^{\prime}, z^{\prime}\right)$ in which we search for the reconstruction frame. An important issue in searching for the reconstruction frame is to find the correct orientation of the invariant $\mathrm{Z}$ axis of the magnetic flux rope. We perform minimum-variance analysis (MVA) on the measured magnetic field vectors (Sonnerup and Cahill, 1967) to obtain three orthogonal directions corresponding to the maximum variance direction, the intermediate variance direction and the minimum variance direction of magnetic field vectors, respectively. The maximum, intermediate and minimum variance direction are taken as the $y^{\prime}, z^{\prime}$ and $x^{\prime}$ axis of the working frame. At the beginning of the analysis the $z^{\prime}$ axis is assumed to be the invariant $Z$ axis of the magnetic flux rope (the initial test $\mathrm{Z}$ axis in the reconstruction frame), as Lepping et al. (1990) have done.
3) Search for the reconstruction frame in the working frame $\left(x^{\prime}, y^{\prime}, z^{\prime}\right)$. The initial test $\mathrm{X}$ axis direction of the reconstruction frame is taken as the projection of $-\boldsymbol{V}_{\boldsymbol{H} \boldsymbol{T}}$ into the plane perpendicular to the initial test $Z$ axis. The initial test $Y$ completes the right-hand orthogonal triad. In this initial reconstruction frame, we integrate the measured $B_{y}$ along the $\mathrm{X}$ axis to obtain $A(x, 0)$ :

$A(x, 0)=\int_{0}^{x} \frac{\partial A}{\partial \varepsilon} d \varepsilon=\int_{0}^{x}-B_{y}(\varepsilon, 0) d \varepsilon$

where $d \varepsilon=-\boldsymbol{V}_{H T} \cdot \boldsymbol{x} d t$ is the distance that the satellite travels during the time interval $d t$. At the same time we compute the transverse pressure $P_{t}(x, 0)=P(x, 0)+B_{z}^{2}(x, 0) / 2 \mu_{0}$ at the corresponding location $x$. Hu et al. (2002) point out that the invariant $\mathrm{Z}$ axis orientation of a magnetic flux rope is determined by finding the direction, for which the data plot of $P_{t}\left(x_{i}, 0\right)$ versus $A_{i}\left(x_{i}, 0\right)$ displays minimal scatter when comparing the plot in the first half of the trajectory (entry into the flux rope) to the plot in the second half of the trajectory (exiting the flux rope). We apply this criteria to evaluate if the invariant $\mathrm{Z}$ axis of the magnetic flux rope (the $\mathrm{Z}$ axis of the reconstruction frame) we found is correct.

4) Beginning with the initial test $Z$ axis of the reconstruction frame in step 2 , we continue to alter the direction of the test $Z$ axis in the hemisphere with $z^{\prime} \geq 0$ in the working frame and repeat the step 3 until we find the correct $Z$ axis of the reconstruction frame satisfying the criteria given in step 3 . In the proper reconstruction frame we compute $P_{t}(x, 0)$ and $A(x, 0)$ and then obtain a function $P_{t}(A)$ at $\mathrm{y}=0$, using the polynomial fitting. The resulting $\operatorname{Pt}(A)$ is then differentiated to obtain $\frac{d}{d A} P_{t}(A)$ at $y=0$. Because the magnetic field lines in the flux rope form the contour plot of $A$, the resulting $\frac{d}{d A} P_{t}(A)$ is applied in the whole transverse section $(\mathrm{X}-\mathrm{Y}$ plane).

5) We then solve the GS equation to obtain the distribution of the magnetic potential A (magnetic field $\boldsymbol{B}$ ) in the transverse section. We choose a rectangular region including the trajectory of the satellite and solve the GS equation using the Taylor expansion in this region:

$$
\begin{aligned}
A(x, y+\Delta y)= & A(x, y)+(\partial A / \partial y)_{x, y}( \pm \Delta y) \\
& +\frac{1}{2}\left(\frac{\partial^{2} A}{\partial y^{2}}\right)_{x, y}( \pm \Delta y)^{2} \\
B_{x}(x, y \pm \Delta y)= & B_{x}(x, y)+\left(\frac{\partial^{2} A}{\partial y^{2}}\right)_{x, y}( \pm \Delta y),
\end{aligned}
$$

where $\quad A(x, y), \quad(\partial A / \partial y)_{x, y}=B_{x} \quad$ and $\frac{\partial^{2} A}{\partial y^{2}}=-\frac{\partial^{2} A}{\partial x^{2}}-\mu_{0} \frac{d}{d A} P_{t}(A)$ are all the known functions of $\mathrm{x}$ at a given $\mathrm{y}$. They can be obtained by the integration from $\mathrm{y}=0$ where all the parameters are measured by Cluster. 


\section{Results and discussion}

During the observation period, the largest distance between the four Cluster satellites $(\mathrm{C} 1, \mathrm{C} 2, \mathrm{C} 3, \mathrm{C} 4)$ is $200 \mathrm{~km}$, so the data from $\mathrm{C} 1, \mathrm{C} 2, \mathrm{C} 3$ and $\mathrm{C} 4$ show almost the same profile. Therefore, the G-S method based on multi-satellite measurements (Sonnerup et al., 2004) is not applied to this case. We only use the data from $\mathrm{C} 1$ to perform the reconstruction. A relatively good constant HT frame velocity $(138.51,-63.85$, $47.34) \mathrm{km} / \mathrm{s}$ is found with the correlation coefficient between $\boldsymbol{V}_{H T} \times \boldsymbol{B}$ and $\boldsymbol{V} \times \boldsymbol{B}$ being $c c=0.931$. Due to the low ion density, the Alfvén velocity is much higher than the plasma velocity in the HT frame, so this case can be investigated with the G-S method. The direction of the invariant $(\mathrm{Z})$-axis of the flux rope in GSE coordinates is $(-0.3892,-0.8591$, 0.3323 ), which is mainly located at the dawn-dusk direction. Furthermore, we get the $\mathrm{X}$ and $\mathrm{Y}$ axis of the reconstruction frame to be directed along $(-0.9129,-0.3118,-0.2632)$ and $(0.1225,-0.4058,-0.9057)$, respectively.

To facilitate understanding the reconstruction results, we rotate the reconstruction frame $180^{\circ}$ around the $\mathrm{X}$ axis to make the $\mathrm{Z}$ axis locate at $(0.3892,0.8591,-0.3323)$, pointing dawnward, and the $\mathrm{Y}$ axis at $(-0.1225,0.4058,0.9057)$, pointing northward. Figure 3 presents the reconstruction results for this case. The northern plasma sheet is at the top of the figure and the Earth is on the left. The closed black curves are the contour plots of the magnetic potential $A$ in the cross-sectional X-Y plane, which are just the magnetic field lines projected onto the cross section. The filled colour inside the curves displays the distribution of the field component $B_{z}$ along the principal axis. The $B_{z}$ value for the different colour is defined by the color bar at the right of the figure. The plus sign at the center of the figure denotes the maximum of the axis field, which is $B_{z}=-12.3 \mathrm{nT}$.

As we can see, $B_{z}$ points toward the inside of the paper and its value decreases gradually from the center to the outer side of the implied flux rope. From the strong core field, which exists inside the flux rope, we can infer that the magnetic flux rope is produced by component reconnection, because anti-parallel reconnection cannot produce a magnetic structure containing a core field like the magnetic flux rope. The black arrows along the $\mathrm{Y}$ axis in the figure are the measured magnetic vectors (not including the axis field $B_{z}$ ). From the directions of the transverse and core fields, we may see that the flux rope is "left-handed". We can also see that the scale length in the $\mathrm{X}$ axis is larger than that in the $\mathrm{Y}$ axis and that the earthward side of the flux rope is broader than the tailward side in the Y direction. The distribution of the magnetic field in the cross section is therefore not exactly axially symmetric, as the ideal model demonstrates. The reason for the shape of this case may be that the flux rope is compressed by the field and plasma in the northern and southern plasma sheet when it moves toward the Earth. The diameter of the flux rope is about $1.5 R_{E}$, so the plasma and magnetic flux carried by this flux rope compared to the whole inner mag-

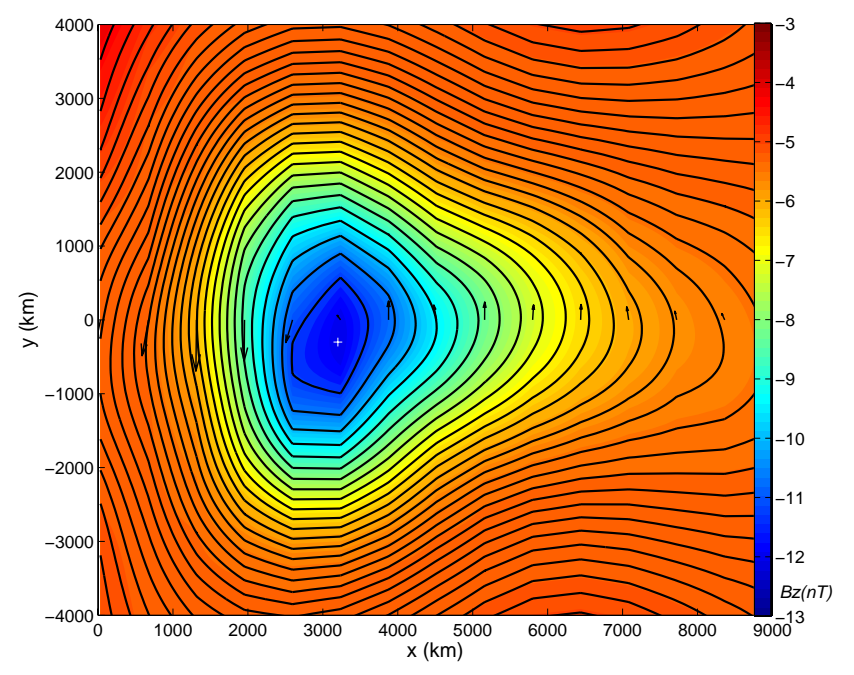

Fig. 3. The reconstruction results of the flux rope on 11 October 2003. The closed black curves are the contour plots of the magnetic potential A on the cross section X-Y plane, which are just the magnetic field lines projected onto the cross section. The filled color inside the curves displays the distribution of the field component $B_{z}$ along the principal axis. The plus at the center of the figure denotes the maximum of the axis field $B_{z}$. The black arrows along the $\mathrm{Y}$ axis in the figure are the measured magnetic vectors (not include the axis field $B_{z}$ ).

netosphere may be insignificant. Nevertheless, the significance of the flux rope in the magnetotail is that its formation indicates the occurrence of simultaneous multi- $\mathrm{X}$ line reconnection in the near-tail.

\section{Summary}

We have applied the Grad-Shafranov technique to reconstruct the magnetic field distribution on the transverse section of a magnetic flux rope $r$ in the magnetotail with no constraint to its geometry. Particularly stable conditions allow us to accurately model the flux tube parameters. We find that the principal axis of the flux rope is almost along the dawn-dusk direction and the diameter of the flux rope is about $1.5 R_{E}$. The magnetic field distribution in the transverse section of the earthward moving flux rope does not correspond to the symmetric circles demonstrated by the ideal model. There is a very strong core field in the flux rope, which may imply that component reconnection is responsible for the formation of this flux rope. The appearance of the flux rope in the magnetic tail can be seen as evidence that multiple- $X$ line reconnection occurs in the magnetotail. The $\mathrm{AE}$ index indicates that there is no substorm occurring ahead of this case, so that multiple-X line reconnection in the plasma sheet does not necessarily appear to trigger the substorm.

Acknowledgements. This work was supported by the National Natural Science Foundation of China Grant No. 40674094, 40621003, 
40125012, 49804009, 4062013094 and 40390150, Ministry of Science and Technology of China Grant 2006CB806305, and International Space Science Institute at Bern, the Hundred Talents Program and International Collaboration Research Team Program of the CAS. We thank the kindly help from Q. Hu. We also thank the Kyoto World Data Center for making the Quick Look Auroral Indices available over the internet.

Topical Editor I. A. Daglis thanks W. J. Hughes for his help in evaluating this paper.

\section{References}

Baker, D. N., Pulkkinen, T. I., Angelopoulos, V., et al.: Neutral line model of substorm: Past results and present view, J. Geophys. Res., 101, 12 975-13 010, 1996.

Balogh, A., Carr, C. M., Acuna, M. H., et al.: The Cluster magnetic field investigation: Overview of in-flight performance and initial results, Ann. Geophys., 19, 1207-1217, 2001, http://www.ann-geophys.net/19/1207/2001/.

deHoffmann, F. and Teller, E.: Magnetohydrodynamic shocks, Phys. Rev. A., 80, 692-703, 1950.

Elphic, R. C., Cattell, C. A., Takahashi, K., et al.: ISEE 1 and 2 Observations of magnetic flux ropes in the magnetotail:FTEs in the plasma sheet?, Geophys. Res. Lett., 13, 648-651, 1986.

Elphic, R. C. and Russell, C. T.: Magnetic flux ropes in the Venus ionosphere: Observations and models, J. Geophys. Res., 88, 5872, 1983.

Hasegawa, H., Sonnerup, B. U. Ö., Dunlop, M. W., et al.: Reconstruction of two-dimensional magnetopause structures from Cluster observations: verification of method, Ann. Geophys., 22, 1251-1266, 2004,

http://www.ann-geophys.net/22/1251/2004/.

Hasegawa, H., Sonnerup, B. U. Ö., Klecker, B., Paschmann, G., Dunlop, M. W., and Rème, H.: Optimal reconstruction of magnetopause structures from Cluster, Ann. Geophys., 23, 973-982, 2005 , http://www.ann-geophys.net/23/973/2005/.

Hau, L.-N. and Sonnerup, B. U. Ö.: Two-dimensional coherent structures in the magnetopause: Recovery of static equilibria from single-spacecraft data, J. Geophys. Res., 104, 6899-6917, 1999.

Hu, Q. and Sonnerup, B. U. Ö.: Reconstruction of magnetic clouds in the solar wind: Orientations and configurations, J. Geophys. Res., 107(A7), 1142, doi:10.1029/2001JA000293, 2002.

Hughes, W. J. and Sibeck, D.: On the 3-dimensional structure of plasmoid, Geophys. Res. Lett., 14, 636-639, 1987.

Johnstone A. D., Alsop, C., Burge, S., et al.: Peace: A Plasma electron and current experiment, Space Sci. Rev., 79, 351-398, 1997.
Khrabrov, A. V. and Sonnerup, B. U. Ö.: DeHofmann-Teller Analysis, Analysis Methods for Multi-Spacecraft Data, edited by: Paschmann G. and Daly, P. W., ISSI Science Report, SR-001, 221-248, 1998.

Kivelson, M. G., Kennel, C. F., McPherron, R. L., et al: The Galileo Earth encounter: Magnetometer and allied measurements, J. Geophys. Res., 98, 11 299-11318, 1993.

Lee, L. C.: A review of magnetic reconnection: MHD models, in: Physics of the Magnetopause, edited by: Song, P., Sonnerup, B. U. Ö., Thomsen, M. F., Geophys Monogr Ser, vol. 90. Washington D.C., AGU, 139-153, 1995.

Lepping, R. P., Jones, J. A., and Burlaga, L. F.: Magnetic field structure of interplanetary magnetic clouds at $1 \mathrm{AU}, \mathrm{J}$. Geophys. Res., 95, 11 957-11 965, 1990.

Moldwin, M. B. and Hughes, W. J.: Geomagnetic substorm association of plasmoid, J. Geophys. Res., 98, 81-88, 1993.

Nishida, A., Scholar, M., Terasawa, T., et al.: Quasi-stagnant plasmoid in the middle tail: A nes preexpansion phaxe phenomenon, J. Geophys. Res., 91, 4245-4255, 1986.

Rème, H., Aoustin, C., Bosqued, J. M., et al.: First multispacecraft ion measurements in and near the Earth's magnetosphere with the identical Cluster ion spectrometry (CIS) experiment, Ann. Geophys., 19, 1303-1354, 2001, http://www.ann-geophys.net/19/1303/2001/.

Schindler, K..: A theory of the substorm mechanism, J. Geophys. Res., 79, 2803-2810, 1974.

Shen, C., Li, X., Dunlop, M., Shi, Q. Q., Liu, Z. X., Lucek, E., and Chen, Z. Q.: Magnetic Field Rotation Analysis and the Applications, J. Geophys. Res., 112(A6), A06211, doi:10.1029/2005JA011584, 2007.

Slavin, J. A., Lepping, R. P., Gjerloev, J., et al.: Geotail observations of magnetic flux ropes in the plasma sheet, J. Geophys. Res., 108(A1), 1015, doi:10.1029/2002JA009557, 2003a.

Slavin, J. A., Lepping, R. P., Gjerloev, J., et al.: Cluster electric current density measurements within a magnetic flux rope in the plasma sheet, Geophys. Res. Lett., 30(7), 1362, doi:10.1029/2002GL016411, 2003b.

Sonnerup, B. U. Ö. and Cahill Jr., L. J.: Magnetopause structure and attitude from Explorer 12 observations, J. Geophys. Res., 72, 171-183, 1967.

Sonnerup, B. U. Ö., Hasegawa, H., and Paschmann, G.: Anatomy of a flux transfer event seen by Cluster, Geophys. Res. Lett., 31, L11803, doi:10.1029/2004GL020134, 2004.

Zhang Yongcun, Liu Zhenxing, Shen Chao, et al.: Double Star TC-1 observation of the earthward flowing plasmoids in the near magnetotail, Cinese. Sci. Bull., 52(13), 1843-1848, 2007. 